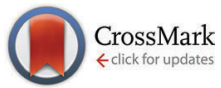

Cite this: J. Mater. Chem. C, 2017, 5, 1255

Received 23rd October 2016, Accepted 12th January 2017

DOI: $10.1039 / c 6 t c 04594 d$

www.rsc.org/MaterialsC

\section{Ultra-high Seebeck coefficient and low thermal conductivity of a centimeter-sized perovskite single crystal acquired by a modified fast growth method $\dagger$}

\author{
Tao Ye, $\ddagger^{\mathrm{ab}}$ Xizu Wang, $\ddagger^{\mathrm{a}}$ Xianqiang Li, ${ }^{\mathrm{c}}$ Alex Qingyu Yan, ${ }^{\mathrm{c}}$ Seeram Ramakrishna* ${ }^{\mathrm{b}}$ \\ and Jianwei $\mathrm{Xu}^{* a}$
}

A centimeter-sized organic-inorganic hybrid lead-based perovskite $\mathrm{CH}_{3} \mathrm{NH}_{3} \mathrm{Pbl}_{3}\left(\mathrm{MAPbl}_{3}\right)$ single crystal was obtained by using a modified fast and inverse-temperature growth method. The optical properties of this single crystal at room and low temperatures were studied in terms of optical absorption and photoluminescence measurements. The single crystal exhibited optical properties with a band-gap of $1.53 \mathrm{eV}$, which is comparable to a reported value. The temperature-dependent UV-vis spectra of this perovskite single crystal showed a unique structural phase transition as the temperatures varied. The thermoelectric properties of this $\mathrm{MAPbl}_{3}$ single crystal were studied, showing that the Seebeck coefficient of $920 \pm 91 \mu \mathrm{V} \mathrm{K} \mathrm{K}^{-1}$ almost remained unchanged from room temperature to $330 \mathrm{~K}$ and it progressively increased with the increase in temperature and reached $1693 \pm 146 \mu \mathrm{V} \mathrm{K} \mathrm{K}^{-1}$ at $351 \mathrm{~K}$. In contrast, there was no very clear trend for thermal conductivities with changes in temperature. The thermal conductivities were maintained between 0.30 and $0.42 \mathrm{~W} \mathrm{~m} \mathrm{~K}^{-1}$ in the temperature range of 298-425 $\mathrm{K}$. These thermoelectric characteristics would be useful for potential thermoelectric applications if the electrical conductivity of this crystal is improved by tuning its composition.

\section{Introduction}

The organic-inorganic hybrid lead-based perovskite $\mathrm{CH}_{3} \mathrm{NH}_{3} \mathrm{PbI}_{3}$ $\left(\mathrm{MAPbI}_{3}\right)$ has recently attracted considerable attention for optoelectronics applications in solar cells and photo-detectors due to its superior characteristics, including a high absorption coefficient, direct band-gap, long carrier lifetime, and high balanced hole and electron mobility, etc. ${ }^{1,2}$ These perovskite materials have emerged as strong candidates for application in photovoltaic and solar cells. In particular, lead halide perovskite $\mathrm{APbB}_{3}$ $(\mathrm{A}=$ methylammonium (MA) and formamidium (FA); $\mathrm{B}=\mathrm{Cl}$, $\mathrm{Br}$ and I) solar cells have reached a record high efficiency of $22.1 \%$ in the past 5 years. ${ }^{3-10}$ Based on the unique properties of these hybrid lead-based perovskites, such as high charge carrier mobility and high diffusion length, they have attracted intense

\footnotetext{
${ }^{a}$ Institute of Materials Research and Engineering (IMRE), Agency for Science, Technology and Research (A*STAR), \#08-03, 2 Fusionopolis Way, Innovis, 138634, Singapore.E-mail:jw-xu@imre.a-star.edu.sg

${ }^{b}$ Department of Mechanical Engineering and Centre of Nanofibers and Nanotechnology (NUSCNN), National University of Singapore, Singapore 117576, Singapore.E-mail: seeram@nus.edu.sg

${ }^{c}$ School of Materials Science and Engineering, Nanyang Technological University, Singapore 639798, Singapore

$\dagger$ Electronic supplementary information (ESI) available. See DOI: 10.1039/c6tc04594d \$ Equal contribution.
}

attention for possible thermoelectric (TE) applications. ${ }^{11-13}$ Low thermal conductivity, and high carrier mobility and Seebeck coefficient are desirable for promising thermoelectric materials. ${ }^{14}$ Compared with inorganic-based thermoelectric materials, polymer and organic-inorganic perovskite materials have attracted more attention for potential thermoelectric application due to their lower thermal conductivity and density. ${ }^{15,16}$ The high scattering parameter at the inorganic-organic interface provided solid evidence for the energy filtering effect, which is in conjunction with the enhancement of the thermal Seebeck coefficient and power factor. ${ }^{17}$ In addition, the enhanced density of states of the energy levels also led to an increase in the Seebeck coefficient in the inorganic-organic material interface. ${ }^{15}$

Thermoelectric materials play a vital role in energy harvesting applications due to their great potential in transforming a temperature gradient into thermoelectricity. ${ }^{18,19}$ The oxide-based thermoelectric materials such as $\mathrm{SrTiO}_{3}, \mathrm{ZnO}, \mathrm{TiO}_{2}, \mathrm{CaMnO}_{3}$, and $\mathrm{Ca}_{3} \mathrm{Co}_{4} \mathrm{O}_{9}$ were fully studied as alternatives to expensive or toxic tellurium-based and selenium-based traditional thermoelectric compounds. ${ }^{20}$ La doped $\mathrm{SrTiO}_{3}$ inorganic perovskites have been developed and their optimized figure-of-merit value of 0.41 (at $973 \mathrm{~K}$ ) for an n-type $\mathrm{Sr}_{1-3 x / 2} \mathrm{La}_{x} \mathrm{TiO}_{3-\delta}$ ceramic with $0.125 \leq x \leq 0.175^{21}$ has been achieved. With the help of advanced $a b$ initio calculations, researchers reported that $\mathrm{MAPbI}_{3}$ exhibits a large value of carrier mobility, which is ascribed to a 
small effective mass and a long diffusion length, while the large value of the Seebeck coefficient is due to the multi-degenerated conduction and valence bands. ${ }^{11,12}$ This intense interest in the lead halide $\mathrm{APbB}_{3}$ perovskites is mostly due to their long electron and hole transport lengths, tunable optoelectronic properties and high absorption coefficients. Meanwhile, these organometallic halide perovskites are low-cost and can be fabricated through facile deposition techniques. ${ }^{22-24}$ Moreover, perovskite single crystals have been shown to possess various merits when compared with their thin film counterparts. ${ }^{25-27}$ More precisely, a remarkably low trap-state density of perovskite single crystals is comparable to that of the best quality silicon in the photovoltaic field and it is five orders of magnitude lower than that of microcrystalline perovskites. Firstly, the carrier mobility in single crystalline perovskites increases with carrier diffusion length. Secondly, the light absorption onset is redshifted, resulting in a large theoretical current density for the crystalline perovskites. As a result, the electrical conductivity and stability of single-crystalline perovskites are expected to be improved. Recently, it was reported that the $\mathrm{MAPbI}_{3}$ single crystal has a low thermal conductivity, which is mainly attributed to the MA rotations in the system. ${ }^{11,12}$ Moreover, an ultralow thermal conductivity of $\sim 0.4 \mathrm{~W} \mathrm{~m} \mathrm{~K}^{-1}$ and a high Seebeck coefficient of $820 \mu \mathrm{V} \mathrm{K}^{-1}$ were also obtained for 3-5 mm sized $\mathrm{MAPbI}_{3}$ crystal drusy facets at a low temperature (from 200 to $300 \mathrm{~K}) .{ }^{13}$ Polycrystals and amorphous perovskite structures, however, cannot be reliably characterized because of their geometries. For instance, the presence of many grain boundaries in polycrystals and amorphous perovskites cannot result in accurate measurement of the thermal conductivity. Therefore, large single crystal perovskites may be necessary to explore their detailed thermoelectric properties.

The growth of perovskite single crystals by using solution crystallization processes always suffers from a slow growth rate. ${ }^{26-31} \mathrm{~A}$ fast crystallization technique - inverse temperature crystallization (ITC) - has been introduced to address the need for fast and massive fabrication of perovskite single crystals. ${ }^{25}$ In our study, we optimized a similar $\mathrm{MAPbI}_{3}$ crystal growth method to obtain a perfect centimeter-sized organic-inorganic hybrid perovskite $\left(\mathrm{MAPbI}_{3}\right)$ single crystal. The growth rate of the resulting single crystal can be further enhanced without compromising its quality. Herein, the physical properties of this single crystal in a wide temperature range have been studied by low-temperature optical absorption and photoluminescence measurements. The thermoelectric behaviour of a $\mathrm{MAPbI}_{3}$ single crystal grown by a fast and solution-based method was examined at above room temperature for the first time. The results exhibited a high and positive Seebeck coefficient in the above room temperature region, thus suggesting that holes are the majority carriers in this kind of $\mathrm{MAPbI}_{3}$ single crystal. In addition, the thermal conductivity of the single crystal was measured and it had a much lower thermal conductivity than reported inorganic perovskites. Apart from the superior photovoltaic characteristics of centimeter-sized perovskite crystals, it revealed unexpected thermoelectric characteristics including a high Seebeck coefficient and low thermal conductivity at room temperature. In addition, it was found that the increase in temperature led to a significant increase in the Seebeck coefficient.

\section{Experimental section}

\section{Materials}

Unless specified otherwise, all materials were purchased from either Alfa Aesar or Sigma-Aldrich. All chemicals were used as purchased without any further purification.

\section{Synthesis of MAI}

Methylammonium iodide was prepared by reacting methylamine, $33 \mathrm{wt} \%$ in ethanol, with hydroiodic acid (HI), $57 \mathrm{wt} \%$ in water, at $0{ }^{\circ} \mathrm{C}$ for $4 \mathrm{~h}$. HI was added dropwise while stirring. Upon drying at $100{ }^{\circ} \mathrm{C}$ on a hotplate to remove the solvent and make methylammonium iodide crystallize, the precipitate was washed with diethyl ether and ethanol three times. The resulting white powder was dried on a hotplate at $65{ }^{\circ} \mathrm{C}$ for $6 \mathrm{~h}$ in air.

Synthesis of the $\mathrm{MAPbI}_{3}$ single crystal and the TE sample. A one molar solution containing $\mathrm{PbI}_{2}$ and MAI was prepared in DMF. The solution was filtered using PTFE filters with $0.2 \mu \mathrm{m}$ pore size. Five milliliters of the filtrate was placed in a vial and the vial was kept in an oil bath undisturbed at $120{ }^{\circ} \mathrm{C}$ for crystal formation. $\mathrm{N}_{2}$ air flow (or using a vacuum pump to keep the pressure in the inner vial at 0.7 atm) was utilized to increase the solvent evaporation and take away the solvent vapour. All the crystal synthesis procedures were carried out under ambient conditions. The crystals used for measurements were grown within 2-3 h. Then, two $100 \mathrm{~nm}$-gold electrodes were deposited on the same surface of the perovskite single crystal with a space distance of $1 \mathrm{~cm}$ through a thermal evaporator within a $\mathrm{N}_{2}$ filled glove box.

\section{Characterization}

A conventional four-point technique was employed to measure the resistivity (MCP-T610, MITSUBISHI CHEMICAL ANALYTECH). The optical absorption spectra were measured on a PerkinElmer Lambda 950UV/VIS/NIR spectrometer. PL spectra were obtained from a triple-grating micro-Raman spectrometer (Horiba-JY T64000). Liquid nitrogen was utilized for cooling the samples. X-ray diffraction experiments were conducted on a Bruker AXS (D8 ADVANCE GADDS) X-ray diffractometer with $\mathrm{Cu} \mathrm{K} \alpha$ radiation $(\lambda=1.54 \AA)$; the beam diameter for this instrument can range from 0.05 to $0.8 \mathrm{~mm}$. The carrier concentration and mobility of the perovskite single crystal were determined using Hall measurements (Bio Rad HL5500) with a four-point-probe using van der Pauw geometry. Four $100 \mathrm{~nm}$-gold electrodes were deposited on the same surface of the perovskite single crystal through a thermal evaporator within a $\mathrm{N}_{2}$ filled glove box. The measurements of the thermal diffusivity $(\kappa)$ were carried out with a Netzsch Microflash system (LFA 457), the density ( $\rho$ ) was calculated from the measured volume and mass of the materials, and the specific heat $\left(C_{\mathrm{p}}\right)$ was measured using a Differential Scanning Calorimetry (DSC, Mettler Toledo) at room temperature. 
The TE properties were measured through a custom-made setup. More precisely, the sample was put on two substrates (as shown in Fig. 3a) with a built-in resistive heater, and the setting temperature can be adjusted by the resistive heaters that were driven by a direct current power supply (PWS2721, Tetronix). The temperature differences of the measuring points were documented by two thermometers (RS 1319A, Taiwan) and the voltage differences at the same points were collected by a Keithley 2400 source meter.

\section{Results and discussion}

The solubility behaviour of MA-based perovskite in $N, N$-dimethylformamide (DMF) is different from common organic-inorganic crystals. It has a high solubility at room temperature, but its solubility decreases with increase in temperature. ${ }^{25}$ This inverse solubility effect can be used to crystallize $\mathrm{MAPbI}_{3}$ rapidly by heating the precursor solution as illustrated in Fig. 1a. A temperature-controllable hot plate was used to heat the oil bath and only a few crystals were formed within the first $10 \mathrm{~min}$. By using $\mathrm{N}_{2}$ air flow (or vacuum) to remove the evaporated DMF solvent promptly, the crystal growth rate can be increased to $\sim 5 \mathrm{~mm}^{3} \mathrm{~h}^{-1}$ for the first hour and the rate would reach $\sim 20 \mathrm{~mm}^{3} \mathrm{~h}^{-1}$ for the following hours. Crystals with a suitable size for thermoelectric measurement (more than $1 \mathrm{~cm}$ in the length direction) can be fabricated within 2-3 h (Fig. 1b). By carefully removing the small crystals from the old solution and placing them in a newly prepared perovskite precursor solution, the crystals can grow further with a large dimension. $\mathrm{X}$-ray diffraction of the perovskite crystal demonstrates high purity as evidenced by similar diffractions of the perovskite $\mathrm{MAPbI}_{3}$ (Fig. 1c) to some previous results. ${ }^{25-27}$ High-resolution XRD was used to examine the quality of the $\mathrm{CH}_{3} \mathrm{NH}_{3} \mathrm{PbI}_{3}$ single crystal. Fig. S1 (ESI $\dagger$ ) shows the $2 \theta$ scan profile with only two very sharp diffractions corresponding to the 200 and 400 planes,
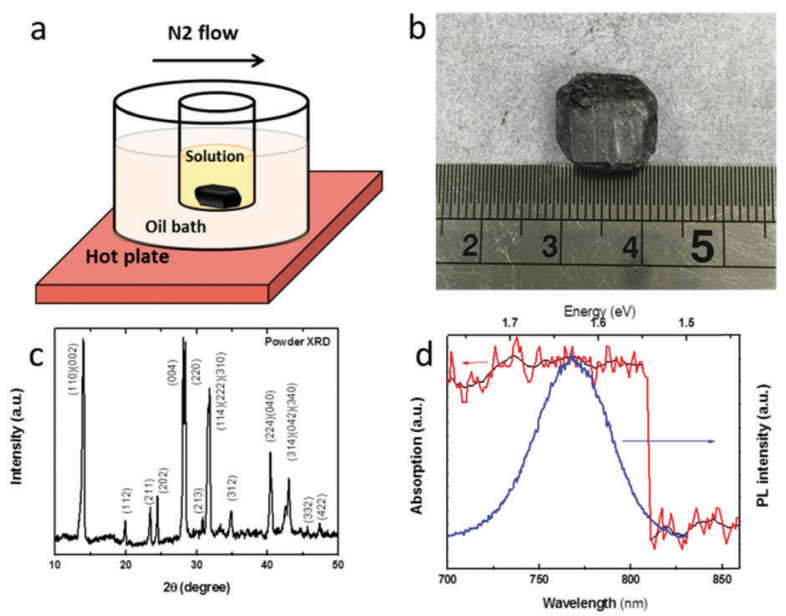

Fig. 1 (a) Schematic graph showing the single crystal growth setup in this study. (b) Image of the $1.3 \mathrm{~cm}$-sized single crystal. (c) X-ray diffraction of the $\mathrm{MAPbl}_{3}$ crystal. (d) Optical absorption (red), smoothed curve (black) and $\mathrm{PL}$ (blue) of a $2 \mathrm{~mm}$-sized $\mathrm{MAPbl}_{3}$ crystal at room temperature. indicating the single-crystalline nature of the sample. ${ }^{27}$ The quality of the crystal grown within a few hours is comparable to those which were grown within several weeks. ${ }^{24}$ The optical absorption and photoluminescence properties of the $\mathrm{MAPbI}_{3}$ single crystal were investigated at room temperature. According to the steady state optical absorption spectrum, it is easy to find a sharp band edge in the spectrum (Fig. 1d). Based on the UV-vis absorption of $\mathrm{MAPbI}_{3}$, its band-gap is estimated to be $1.53 \mathrm{eV}$. The magnitude of the band-gap of the $\mathrm{MAPbI}_{3}$ single crystal grown by using the $\mathrm{N}_{2}$ flow-assisted ITC method is in good agreement with that of the single crystals grown by an ITC or anti-solvent crystallization method at room temperature. ${ }^{25,28}$ The narrow and blue-shifted PL peak $(\sim 65 \mathrm{~nm})$ indicates a low trap density in the $\mathrm{MAPbI}_{3}$ crystal and the PL peak width was determined from the intensity difference crossing the first derivative of the PL spectrum. The excellent overlapping of the PL spectrum with the optical absorption spectrum of the $\mathrm{MAPbI}_{3}$ single crystal allows photon recycling in the thick crystal by reabsorbing the emission, which is beneficial for solar thermoelectric generation, an emerging technology combining concentrated solar power and thermoelectric effects (the corresponding optical properties of the $\mathrm{MAPbI}_{3}$ thin film can be found in Fig. S2, ESI $\dagger){ }^{13}$

Original single crystals instead of powder (mechanically fabricated from single crystals) $)^{25-28}$ were used for this experiment. To study the ratio of free charges over the total photo-excitation in this single crystal, temperature-dependent optical absorption spectra are recorded from $340 \mathrm{~K}$ to $77 \mathrm{~K} .^{32}$ The absorption edge (valance band maximum) of the $\mathrm{MAPbI}_{3}$ single crystal is $1.53 \mathrm{eV}$. Generally, the tetragonal phase of $\mathrm{MAPbI}_{3}$, in which the methylammonium moiety is disordered, exists from $160 \mathrm{~K}$ to room temperature. In contrast, the orthorhombic phase, in which the methylammonium unit is ordered, exists below $160 \mathrm{~K}^{29,32}$ As can be seen from Fig. 2a, the spectra of the $\mathrm{MAPbI}_{3}$ single crystal show a remarkable structural phase transition during the cooling period. More precisely, there is a significant shift of the absorption edge through $170-210 \mathrm{~K}$ in a single crystal. This behaviour can be regarded as a crystal phase transition of the perovskite single crystal from a tetragonal to an orthorhombic structure at $\sim 170 \mathrm{~K}$, inducing a blue shift in the $\mathrm{MAPbI}_{3}$ material absorption edge of $\sim 34 \mathrm{meV}$. As the temperature decreases, the absorption band edges of the sample show blue shifts called the 'Varshni' trend (a positive thermal expansion coefficient), which has been observed in many lead composite semiconductors. ${ }^{32-34}$ Different from the perovskite thin film, no typical exciton peak was observed in the wide temperature range (Fig. 2a), thus implying that the exciton binding energy of this single crystal is much less than that in the film, resulting in a high free carrier generation ratio within the crystal.

We also present a temperature-dependent study of PL emission of the perovskite single crystal to explore the nature of recombination channels within the $\mathrm{MAPbI}_{3}$ single crystal at a wide temperature range. ${ }^{35,36}$ Fig. $2 \mathrm{~b}$ shows the PL spectra at a wide temperature range from $77 \mathrm{~K}$ to $340 \mathrm{~K}$ with laser excitation at $532 \mathrm{~nm}$. The PL spectral profile is independent of the excited laser intensity and photon energy of the excitation in this study. 


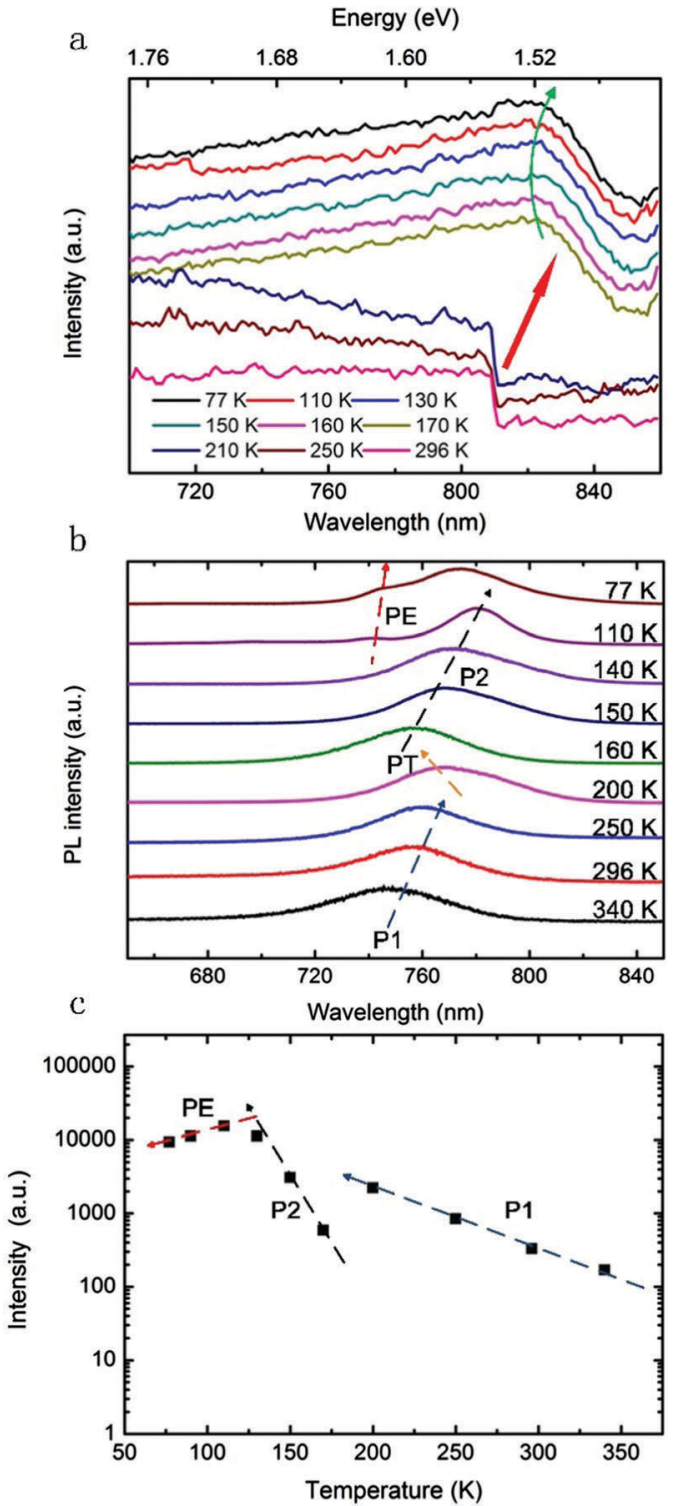

Fig. 2 Optical results of the $\mathrm{MAPb}_{3}$ single crystal. (a) Temperaturedependent optical absorption spectra of the single crystal at different temperatures; (b) temperature-dependent PL spectra of the single crystal at different temperatures; (c) the $\mathrm{PL}$ intensity of the $\mathrm{MAPb}_{3}$ single crystal vs. temperature. P: process, PT: phase transition and PE: process with abnormal exciton behaviour.

At room temperature, a broad peak at $\sim 1.62 \mathrm{eV}$ can be seen in the PL spectrum. The PL intensity shows a rapid increase by more than one order of magnitude when the temperature decreases to $200 \mathrm{~K}$ and the peak position red-shifts to $\sim 1.61 \mathrm{eV}$ (P1 of Fig. 2c). According to a previous work, the estimated $\mathrm{MAPbI}_{3}$ band-gap energy is $1.61 \mathrm{eV} ;{ }^{35}$ only a very small Stokes shift occurs here, and thus the PL of the single crystal is generated from radiative recombination [conduction band (electrons) to valence band (holes)]. At $T \leq 160 \mathrm{~K}$, a new blue-shifted PL peak appears at $\sim 1.64 \mathrm{eV}$, and it grows rapidly as the temperature decreases (one order of magnitude in a $20 \mathrm{~K}$ temperature range) and becomes the main feature at $77 \mathrm{~K}$ (P2 of Fig. 2c). The evolution of the PL spectrum of the single crystal with decreasing temperature is due to a tetragonal-to-orthorhombic phase transition, in good agreement with the optical absorption result. The tetragonal phase at high temperature with the PL peak at $1.61-1.64 \mathrm{eV}$ is ascribed to the collective rotation (around the $c$-axis) of each $\mathrm{PbI}_{6}$ octahedron from its symmetric position in the cubic structure. In contrast, the low temperature phase (orthorhombic) with a PL peak at 1.59-1.64 eV is derived from the tilting of the $\mathrm{PbI}_{6}$ octahedra out of the $a b$ plane. ${ }^{36-38}$ Since the orthorhombic $\mathrm{MAPbI}_{3}$ is a direct-band-gap crystal, ${ }^{39-41}$ multiple PL peaks indicate the coexistence of the tetragonal and orthorhombic phases of $\mathrm{MAPbI}_{3}$ in the temperature range of $200-160 \mathrm{~K}$. When the temperature continues to go down to $110 \mathrm{~K}$, a new PL peak appears at $1.67 \mathrm{eV}$, which may originate from free excitons and donor-acceptor-pair transitions. A peak intensity decrease can be observed in this period (PE of Fig. 2c) as the new peak enhances with a decrease in temperature. Further modelling and experiments are needed to understand the inter-conversion among the thermal activation of the trap states into band-gap excitons, the broad distribution of the trap states, and the competition between the radiative and non-radiative recombination rates of these states. ${ }^{36,42}$

The Hall effect is another important tool to examine the quality of the perovskite single crystal. ${ }^{26,27}$ It was found that the $\mathrm{MAPbI}_{3}$ single crystal shows a high carrier mobility of $59 \mathrm{~cm}^{2} \mathrm{~V}^{-1} \mathrm{~s}^{-1}$ and a free hole concentration of $4.72 \times 10^{11} \mathrm{~cm}^{-3}$. The thermoelectric properties of the perovskite single crystal were measured with a custom-made setup. Specifically, a voltage controllable DC power supply and electric resistance elements were used to create a temperature gradient, and two thermometers together with two copper points (wires) were used to measure the temperature gradient. The voltage difference at the same points was collected by another two copper points (wires) and a Keithley 2400 (Fig. 3a). Plots of thermal voltage $v s . \Delta T$ measured at room temperature $\left(25{ }^{\circ} \mathrm{C}\right)$ and the Seebeck coefficients obtained at different average temperatures are shown in Fig. $3 \mathrm{~b}$ and c, respectively.
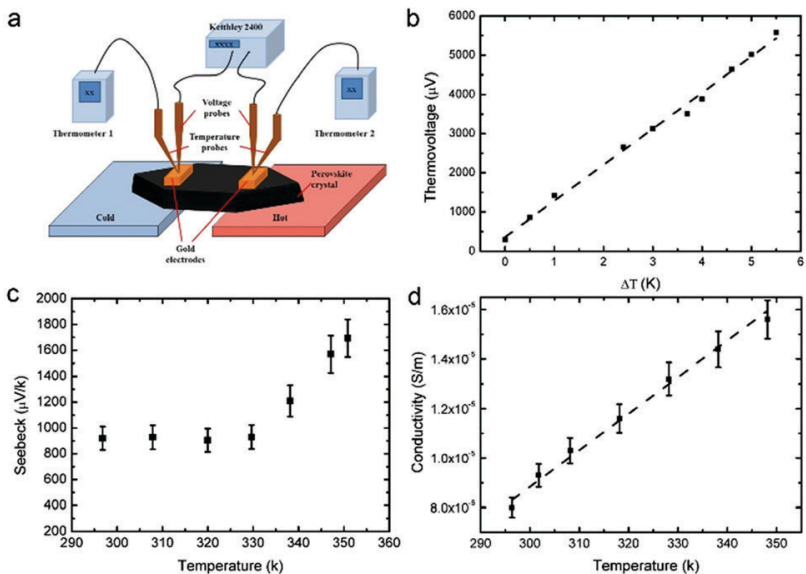

Fig. 3 Thermoelectric property characterization of the perovskite single crystal. (a) Schematic graph of the thermoelectric property measurement. (b) Thermal voltages at different applied temperature differences. The Seebeck coefficient can be obtained from the linear fit (dashed lines); (c) Seebeck coefficients at different average temperatures; (d) conductivity at different temperatures and the dashed line is the linear fit. 
The positive Seebeck coefficient indicates that free holes are the majority carrier type in the $\mathrm{MAPbI}_{3}$ single crystal, which is consistent with the Hall effect measurement, but the nonmonotonic temperature dependency of the Seebeck coefficient indicates that electrons also participate in the charge transport, and the temperature-dependent mobility of the electrons is different from the one of the holes. ${ }^{13,29}$ Moreover, the measured Seebeck coefficient value of $920 \pm 91 \mu \mathrm{V} \mathrm{K}^{-1}$ for this perovskite single crystal at room temperature $(297 \mathrm{~K})$ is higher than that $\left(820 \mu \mathrm{V} \mathrm{K}^{-1}\right.$ and or $\left.\sim 300 \mu \mathrm{V} \mathrm{K}^{-1}\right)$ of the crystal facets made with a slow solvent evaporation method. ${ }^{13,29}$ The trend in Fig. 3c suggests that it could reach an even larger value of $1693 \pm 146 \mu \mathrm{V} \mathrm{K}^{-1}$ at $351 \mathrm{~K}$, which is 5.4-33.9 times that in the pure and La-doped $\mathrm{SrTiO}_{3}$ inorganic perovskites. ${ }^{20}$ This result seems to conclude that with the temperature rise, the enhanced high energy carrier transport and increased density of states of the energy levels within the single crystal perovskite lead to the improved Seebeck coefficient. ${ }^{15,17,43,44}$ The electrical conductivity of the single crystal was also measured in the temperature range of 296 to $350 \mathrm{~K}$ as shown in Fig. 3d. The conductivity increases with the increase of temperature, which is consistent with a temperature-activated transport thermal conductivity $\sim \exp \left(E_{\mathrm{a}} / T\right)$ with an energy barrier of $128 \mathrm{meV}$ (Fig. S3, ESI $\dagger)^{45,46}$

A low thermal conductivity of around $0.30-0.42 \mathrm{~W} \mathrm{~m} \mathrm{~K}^{-1}$ in the temperature range of 299 to $424 \mathrm{~K}$ was observed for this perovskite single crystal (Fig. 4), and this value was much smaller than that of the pure or La doped $\mathrm{SrTiO}_{3}$ perovskites (4.5-7 $\left.\mathrm{W} \mathrm{m} \mathrm{K}^{-1}\right) .{ }^{20}$ Apart from the electrical conductivity, an ultrahigh Seebeck coefficient and low thermal conductivity were observed in this centimeter-sized perovskite single crystal, suggesting that the enhancement of electrical conductivity would be the key factor to achieve high thermoelectric performance of perovskite materials. Further increases in electrical conductivity can be achieved by doping some other metallic elements that can form more covalent bonds with the iodine ions in the perovskite crystal. ${ }^{47-49}$ It is noteworthy that the electrical conductivity is increased by 10 orders of magnitude in MASnI $_{3}$ with a value of $10^{5} \mathrm{~S} \mathrm{~m}^{-1}$. ${ }^{13}$ It shows a path towards significant improvements in the electrical conductivity of MA based perovskites. Holes are the majority carrier type for the $\mathrm{MAPbI}_{3}$ single crystal, but electrons also participate in the

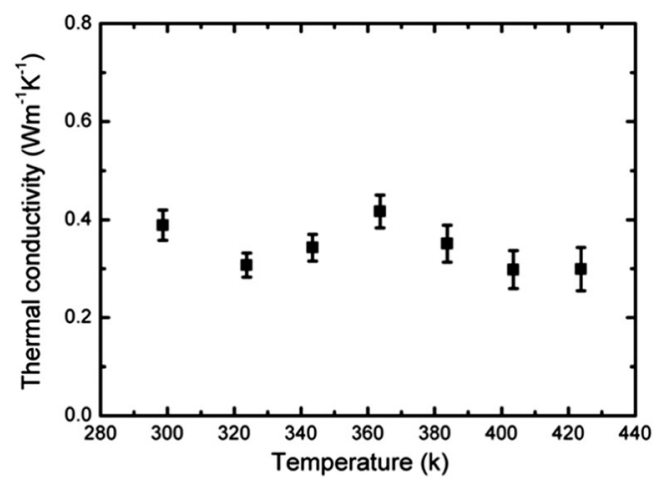

Fig. 4 Measured thermal conductivity as a function of the average temperature. charge transport and thus influence the overall thermoelectric performance. This phenomenon is also called amphoterism, ${ }^{50}$ which should be avoided in thermoelectric materials/applications. Therefore, improvement in the thermoelectric performance of the $\mathrm{MAPbI}_{3}$ single crystal could be achieved by suppressing the amphoterism effect through heavy p-doping of this material.

\section{Conclusions}

In conclusion, a large $\mathrm{MAPbI}_{3}$ single crystal was grown using a modified fast growth method, and the Seebeck coefficient, electrical conductivity and thermal conductivity of the $\mathrm{MAPbI}_{3}$ single crystal were measured. The Seebeck coefficients remained roughly constant in the temperature range of 298-330 K, and then significantly increased with the increase in temperature. A large and positive Seebeck coefficient of $920 \pm 91 \mu \mathrm{V} \mathrm{K}^{-1}$ at room temperature and an ultra-high value of $1693 \pm 146 \mu \mathrm{V} \mathrm{K} \mathrm{K}^{-1}$ at $351 \mathrm{~K}$ were observed. A low thermal conductivity of $0.30-0.42 \mathrm{~W} \mathrm{~m} \mathrm{~K} \mathrm{~K}^{-1}$, which is comparable to that of polymer materials, is obtained. It is envisioned that the $\mathrm{MAPbI}_{3}$ single crystal could be considered as a new class of thermoelectric material if proper elemental doping can be incorporated to improve the electrical conductivity and in turn increase the overall figure-of-merit value.

\section{Acknowledgements}

The authors acknowledge financial support from the A*STAR, Industry Alignment Fund, Pharos "Hybrid thermoelectric materials for ambient applications" Programme (Grant No. 1527200021). This work is also supported by the Grantor Lloyd's Register Foundation (R-265-000-553-597) and T. Y. acknowledges the National University of Singapore for the research scholarship. The authors thank Mr Xingzhi Wang from the Nanyang Technological University for assisting with the experiments.

\section{Notes and references}

1 S. D. Stranks, G. E. Eperon, G. Grancini, C. Menelaou, M. J. P. Alcocer, T. Leijtens, L. M. Herz, A. Petrozza and H. J. Snaith, Science, 2013, 342, 341.

2 G. Xing, N. Mathews, S. Sun, S. S. Lim, Y. M. Lam, M. Gräetzel, S. Mhaisalkar and T. C. Sum, Science, 2013, 342, 344.

3 National Renewable Energy Laboratory (NREL), Research Cell Efficiency Records, http://www.nrel.gov/ncpv/images/ efficiency_chart.jpg, accessed: December, 2016.

4 A. Kojima, K. Teshima, Y. Shirai and T. Miyasaka, J. Am. Chem. Soc., 2009, 131, 6050.

5 N. J. Jeon, J. H. Noh, W. S. Yang, Y. C. Kim, S. Ryu, J. Seo and S. I. Seok, Nature, 2015, 517, 476.

6 W. Chen, Y. Wu, Y. Yue, J. Liu, W. Zhang, X. Yang, H. Chen, E. Bi, I. Ashraful, M. Graetzel and L. Han, Science, 2015, 350, 944.

7 B. Saparov and D. B. Mitzi, Chem. Rev., 2016, 116, 4558. 
8 S. Pang, H. Hu, J. Zhang, S. Lv, Y. Yu, F. Wei, T. Qin, H. Xu, Z. Liu and G. Cui, Chem. Mater., 2014, 26, 1485.

9 J. Chen, S. Zhou, S. Jin, H. Li and T. Zhai, J. Mater. Chem. C, 2016, 4, 11.

10 Q. Wang, H. Chen, G. Liu and L. Wang, Sci. Bull., 2015, 60, 405.

11 Y. He and G. Galli, Chem. Mater., 2014, 26, 5394.

12 T. Hata, G. Giorgi and K. Yamashita, Nano Lett., 2016, 16, 2749.

13 X. Mettan, R. Pisoni, P. Matus, A. Pisoni, J. Jaćimović, B. Náfrádi, M. Spina, D. Pavuna, L. Forro and E. Horváth, J. Phys. Chem. C, 2015, 119, 11506.

14 K. F. Hsu, S. Loo, F. Guo, W. Chen, J. S. Dyck, C. Uher, T. Hogan, E. K. Polychroniadis and M. G. Kanatzidis, Science, 2004, 303, 818.

15 B. Russ, A. Glaudell, J. J. Urban, M. L. Chabinyc and A. S. Rachel, Nat. Rev. Mater., 2016, 1, 16050.

16 A. Pisoni, J. Jaćimović, O. S. Barišić, M. Spina, R. Gaál, F. László and E. Horváth, J. Phys. Chem. Lett., 2014, 5, 2488.

17 M. He, J. Ge, Z. Lin, X. Feng, X. Wang, H. Lu, Y. Yang and F. Qiu, Energy Environ. Sci., 2012, 5, 8351.

18 G. J. Snyder and E. S. Toberer, Nat. Mater., 2008, 7, 105-114.

19 L. E. Bell, Science, 2008, 321, 1457.

20 Z. Lu, H. Zhang, W. Lei, D. C. Sinclair and I. M. Reaney, Chem. Mater., 2016, 28, 925.

21 Y. Lin, C. Norman, D. Srivastava, F. Azough, L. Wang, M. Robbins, K. Simpson and I. A. Kinloch, ACS Appl. Mater. Interfaces, 2015, 7, 15898.

22 J. H. Noh, S. H. Im, J. H. Heo, T. N. Mandal and S. I. Seok, Nano Lett., 2013, 13, 1764.

23 M. R. Filip, G. E. Eperon, H. J. Snaith and F. Giustino, Nat. Commun., 2014, 5, 5757.

24 V. D'Innocenzo, A. R. Srimath Kandada, M. De Bastiani, M. Gandini and A. Petrozza, J. Am. Chem. Soc., 2014, 136, 17730.

25 M. I. Saidaminov, A. L. Abdelhady, B. Murali, E. Alarousu, V. M. Burlakov, W. Peng, I. Dursun, L. Wang, Y. He, G. Maculan, A. Goriely, T. Wu, O. F. Mohammed and O. M. Bakr, Nat. Commun., 2015, 6, 7586.

26 Q. Dong, Y. Fang, Y. Shao, P. Mulligan, J. Qiu, L. Cao and J. Huang, Science, 2015, 347, 967.

27 Y. Liu, Z. Yang, D. Cui, X. Ren, J. Sun, X. Liu, J. Zhang, Q. Wei, H. Fan, F. Yu, X. Zhang, C. Zhao and S. Liu, Adv. Mater., 2015, 27, 5176.

28 D. Shi, V. Adinolfi, R. Comin, M. Yuan, E. Alarousu, A. Buin, Y. Chen, S. Hoogland, A. Rothenberger, K. Katsiev, Y. Losovyj, X. Zhang, P. A. Dowben, O. F. Mohammed, E. H. Sargent and O. M. Bakr, Science, 2015, 347, 519.
29 C. C. Stoumpos, C. D. Malliakas and M. G. Kanatzidis, Inorg. Chem., 2013, 52, 9019.

30 Y. Dang, Y. Liu, Y. Sun, D. Yuan, X. Liu, W. Lu, G. Liu, H. Xia and X. Tao, CrystEngComm, 2015, 17, 665.

31 T. Baikie, Y. Fang, J. M. Kadro, M. Schreyer, F. Wei, S. G. Mhaisalkar, M. Graetzel and T. J. White, J. Mater. Chem. A, 2013, 1, 5628.

32 V. D’Innocenzo, G. Grancini, M. J. Alcocer, A. R. S. Kandada, S. D. Stranks, M. M. Lee, G. Lanzani, H. J. Snaith and A. Petrozza, Nat. Commun., 2014, 5, 3586.

33 Y. P. Varshni, Physica, 1967, 34, 149.

34 T. Ye, X. Jiang, D. Wan, X. Wang, J. Xing, T. Venkatesan, Q. Xiong and S. Ramakrishna, ChemPhysChem, 2016, 17, 1.

35 W. Kong, Z. Ye, Z. Qi, B. Zhang, M. Wang, A. Rahimi-Iman and H. Wu, Phys. Chem. Chem. Phys., 2015, 17, 16405.

36 X. Wu, M. T. Trinh, D. Niesner, H. Zhu, Z. Norman, J. S. Owen, O. Yaffe, B. J. Kudisch and X. Y. Zhu, J. Am. Chem. Soc., 2015, 137, 2089.

37 Y. Kawamura, H. Mashiyama and K. Hasebe, J. Phys. Soc. Jpn., 2002, 71, 1694.

38 C. Wehrenfennig, M. Liu, H. J. Snaith, M. B. Johnston and L. M. Herz, APL Mater., 2014, 2, 081513.

39 F. Brivio, A. B. Walker and A. Walsh, APL Mater., 2013, 1, 042111.

40 Y. Wang, T. Gould, J. F. Dobson, H. Zhang, H. Yang, X. Yao and H. Zhao, Phys. Chem. Chem. Phys., 2014, 16, 1424.

41 W.-J. Yin, T. Shi and Y. Yan, Appl. Phys. Lett., 2014, 104, 063903.

42 S. D. Stranks, V. M. Burlakov, T. Leijtens, J. M. Ball, A. Goriely and H. J. Snaith, Phys. Rev. Appl., 2014, 2, 034007.

43 C. J. Vineis, A. Shakouri, A. Majumdar and M. G. Kanatzidis, Adv. Mater., 2010, 22, 3970.

44 A. Shakouri, Annu. Rev. Mater. Res., 2011, 41, 399.

45 A. A. Talin, A. Centrone, A. C. Ford, M. E. Foster, V. Stavila, P. Haney, R. A. Kinney, V. Szalai, F. El Gabaly, H. P. Yoon and F. Léonard, Science, 2014, 343, 66.

46 K. J. Erickson, F. Léonard, V. Stavila, M. E. Foster, C. D. Spataru, R. E. Jones, B. M. Foley, P. E. Hopkins, M. D. Allendorf and A. A. Talin, Adv. Mater., 2015, 27, 3453.

47 S. Pantelides, Rev. Mod. Phys., 1978, 50, 797.

48 D. Emin, Phys. Rev. B: Condens. Matter Mater. Phys., 1999, 59, 6205.

49 T. Devic, M. Evain, Y. Moelo, E. Canadell, P. Auban-Senzier, M. Fourmigue and P. Batail, J. Am. Chem. Soc., 2003, 125, 3295.

50 Y. Qiu, L. Xi, X. Shi, P. Qiu, W. Zhang, L. Chen, J. R. Salvador, J. Y. Cho, J. Yang, Y. C. Chien, S. Chen, Y. Tang and G. J. Snyder, Adv. Funct. Mater., 2013, 23, 3194. 